\title{
L'indépendance de la régulation des activités ferroviaires en France : la quadrature du cercle
}

\author{
Corinne Blanquart ${ }^{a}$ et Alexandra Hyard ${ }^{b *}$ \\ a Directrice de recherches, SPLOTT, IFSTTAR, France \\ ${ }^{b}$ Maître de conférences, CLERSE, Faculté des Sciences Economiques et Sociales, \\ Université Lille 1 - 59655 Villeneuve d'Ascq
}

\section{Résumé}

La libéralisation des activités ferroviaires a favorisé autant l'émergence de nouveaux opérateurs privés que celle de nouvelles structures publiques de régulation et de contrôle. L'une des difficultés majeures de ces structures réside dans leur indépendance, à la fois, par rapport aux acteurs du secteur, mais aussi par rapport à l'État, historiquement impliqué dans le secteur ferroviaire. Les fonctions essentiellement techniques des structures de régulation peuvent-elles s'exercer indépendamment des acteurs du secteur, et notamment des acteurs historiques ? L'évolution des modalités de régulation du secteur ferroviaire en France, depuis la Mission de Contrôle des Activités Ferroviaires jusqu'à la récente Autorité de Régulation des Activités Ferroviaires, traduit cette difficulté. Pour rendre davantage effective l'indépendance de cette Autorité, la France a choisi de lui conférer des pouvoirs plus conséquents, au prix cependant de contre-pouvoirs insuffisants.

(c) 2012 IDMP/Lavoisier SAS. Tous droits réservés

Mots clés : Activités ferroviaires, indépendance, France, libéralisation, Union européenne, régulation.

\section{Abstract}

The independance of the rail operations regulation in France: the squared circle. The liberalization of railway operations has encouraged the emergence of both new private operators and new public structures of regulation and control. One of the major difficulties of these structures is their independence from both the rail-sector actors and the State that has a long tradition of intervention in this sector. Can the essentially technical functions of these regulatory structures be applicable independently of the rail-sector actors and in particular of the long established actors? The change of regulation methods in the French railway sector, beginning with the creation of the "Mission de Contrôle

*Auteur correspondant : alexandra.hyard@univ-lille1.fr doi:10.3166/pmp.29.591-606 @ 2012 IDMP/Lavoisier SAS. Tous droits réservés 
des Activités Ferroviaires" and continuing with the new "Autorité de Régulation des Activités Ferroviaires", reflects this difficulty. To enforce the independence of this Authority, the French government has chosen to award it stronger powers, without establishing adequate checks and balances.

Keywords: European Union, independence, France, liberalization, rail operations, regulation.

\section{Introduction}

Le 5 juillet 2010, une tribune du journal Le Monde, rédigée par Philippe Aghion et Julia Cage, appelait au « retour de l'État régulateur». Ce retour est, selon les auteurs, nécessaire concernant les trois fonctions étatiques suivantes : «1/ celle de régulateur et de garant des contre-pouvoirs démocratiques ...; 2/ celle de redistributeur et de garant du contrat social ... ; 3/ celle d'investisseur dans l'innovation, la formation et le savoir » (Aghion et Cage, $2010: 1$ ). Qu'en est-il du rôle de l'État dans le transport et, plus particulièrement, dans le transport ferroviaire ? L'ouverture à la concurrence de ces activités de réseau, historiquement organisées sous la forme d'un monopole public, s'est accompagnée de la mise en place d'instances de régulation, dont l'indépendance est gage d'efficacité (Lévêque, 2004). La mise en place de telles instances de régulation est souhaitée par l'Union européenne afin d'assurer un accès équitable et non discriminatoire aux réseaux et aux services ferroviaires. Cette indépendance soulève cependant un problème : comment assurer la coexistence au sein de l'État des fonctions d'actionnaire et de tuteur d'entreprises publiques qui incombent au gouvernement et celles de régulateur neutre vis-à-vis de l'ensemble des entreprises ?

La question, qui se pose, est donc celle de la transformation du rôle de l'État dans les activités ferroviaires au moment où l'organisation monopolistique et intégrée de ces dernières est remise en cause (Savy, 2010). En effet, comme le rappelle Genoud (2004 : 188), la libéralisation des industries de réseau comme le transport ferroviaire ne signifie pas « une réduction quantitative de l'intervention publique, mais plutôt sa transformation ». Cette transformation est-elle suffisante pour faire face aux enjeux d'indépendance associés à la régulation?

Avant d'aller plus en avant dans l'analyse, un point de terminologie doit être éclairci. Le terme « régulation » fait, en effet, l'objet d'une interprétation ambiguë en raison d'acceptions différentes en français et en anglais. En français, la régulation a une portée très générale car elle désigne le fait de maintenir en équilibre, d'assurer le fonctionnement correct d'un système. Elle ne doit alors pas être confondue avec la réglementation, qui est le fait d'assujettir à un règlement, c'est-à-dire à un ensemble ordonné de règles. Par conséquent, la réglementation ne peut être assimilée à la régulation puisqu'elle n'est que l'une des forces à l'œuvre au sein de la régulation. Or, le terme anglais « regulation $~^{1}{ }^{1}$ renvoie pour sa part principalement à la réglementation.

Cette ambiguïté se retrouve notamment dans le nom de l'instance de régulation récemment

\footnotetext{
${ }^{1}$ Rappelons que ce substantif vient du verbe « to regulate » qui signifie « to control by rule, to direct ».
} 
créée en France : l'Autorité de Régulation des Activités Ferroviaires (ARAF). Comme la loi 1503 du 8 décembre $2009^{2}$ l'indique, cette autorité « concourt au bon fonctionnement du service public et des activités concurrentielles de transport ferroviaire » (art. 11) mais aussi « est consultée sur les projets de textes réglementaires relatifs à l'accès au réseau ferroviaire, à la conception, la réalisation et l'utilisation des infrastructures et des matériels de transport ferroviaire » (art. 15-VIII).

L'objectif de cet article est d'éclairer non pas la politique de libéralisation des activités ferroviaires menée par l'Union européenne dans sa globalité - même si, à la différence par exemple de la libéralisation européenne du transport aérien ${ }^{3}$, elle a finalement été peu étudiée - mais plutôt un aspect de cette politique : celui de la régulation. Plus précisément encore, il s'intéressera à la création en France de l'ARAF. Cette création soulève, en effet, plusieurs questions : quelles sont les missions de l'ARAF ? En quoi cette instance se distingue-t-elle de la précédente instance chargée du contrôle ferroviaire en France ? L'ARAF jouit-elle d'une réelle indépendance ? Enfin, ses modalités de constitution et de fonctionnement traduisent-elles une évolution suffisante de l'intervention de l'État pour assurer l'indépendance de cette instance?

L'hypothèse que nous testerons est qu'en dépit des réformes engagées par la Commission européenne afin de faire évoluer le rôle de l'État dans les activités ferroviaires et des gardefous qui entourent l'ARAF, cette instance aux pouvoirs importants risque d'être «capturée » par les intérêts privés qu'elle est censée réguler mais aussi, par les intérêts publics dont elle émane ${ }^{4}$. Ce risque n'est, bien sûr, pas encore pleinement avéré concernant l'ARAF qui n'est opérationnelle que depuis le $1^{\text {er }}$ décembre 2010. Mais, comme nous le verrons, certains éléments relatifs à la constitution même de l'ARAF laissent présager cette « capture ».

En raison de la création récente de l'instance, l'analyse se basera sur la lecture des textes juridiques relatifs à cette instance. Le déterminisme du cadre juridique sur le fonctionnement de l'instance sera nuancé par l'analyse de ses décisions et par l'évaluation a posteriori du fonctionnement de l'instance précédente, la MCAF. Cette lecture sera, en outre, illustrée par quelques exemples tirés de l'actualité.

L'article s'articule autour de trois parties. La première partie reviendra sur le principe de la régulation et sur les conditions de sa réussite. La deuxième partie dressera le bilan de la mise en œuvre de la première instance de régulation en matière ferroviaire. La troisième partie soulignera les limites, pour certaines encore potentielles, de l'ARAF.

\section{Libéralisation et nouvelles formes de régulation publique}

Lorsqu'elle est concomitante à la libéralisation ${ }^{5}$, la régulation consiste à organiser l'ouverture à la concurrence de services en réseaux autrefois fournis par des entreprises (le plus souvent publiques) dans le cadre de monopoles réglementés par l'État.

\footnotetext{
${ }^{2}$ Loi n²009-1503 du 8 décembre 2009 relative à l'organisation et à la régulation des transports ferroviaires et portant diverses dispositions relatives aux transports, JORF nº 0285 du 9 décembre 2009.

${ }^{3}$ Voir, par exemple, OCDE (1988), Reynolds-Feighan (1992), Touwaide (2006).

${ }^{4}$ En ceci, elle se rapproche des régulateurs français du transport aérien (voir Landier et Thesmar, 2010 : 91 ).

${ }^{5}$ Nous ne ferons pas ici référence à la régulation comme encadrement de secteurs sujets à des risques comme le secteur financier.
} 


\subsection{La notion de régulation}

Le rapport dirigé par Bergougnoux (2000 : 206), pour le Commissariat Général du Plan, concernant les services publics en réseaux, définit la régulation comme «l'ensemble des interventions des pouvoirs publics visant à instaurer la concurrence autant qu'il est nécessaire dans un secteur où elle n'existait pas ou très peu, et à concilier l'exercice loyal de cette concurrence avec les missions d'intérêt général dont sont investis les services publics en réseaux».

La régulation s'avère alors nécessaire au regard de deux éléments :

- la coexistence aujourd'hui dans un même secteur de segments d'activité présentant d'une part les caractéristiques d'un monopole naturel, comme cela est le cas pour les infrastructures ferroviaires et supportant par ailleurs un certain nombre de services (l'exploitation ferroviaire par exemple) ;

- par rapport à ces services, la mise en œuvre de missions d'intérêt général assurées par un seul opérateur et d'activités en situation concurrentielle nécessite une action publique spécifique qui parvienne à concilier ces deux principes apparemment contradictoires. La régulation recouvre, en définitive, deux fonctions :

- l'introduction et le maintien de la concurrence (la régulation économique) ; il s'agit d'organiser l'ouverture à la concurrence, mais aussi de veiller à ce que n'apparaissent pas des comportements anticoncurrentiels;

- la mise en œuvre de politiques publiques relatives aux missions de service public (la régulation socio-politique) ou fixant des objectifs plus larges (le transport durable, l'aménagement du territoire...).

Genoud (2004:190) distingue, pour sa part, trois types de régulation, recouvrant cinq fonctions (tableau 1).

La langue française distingue la régulation de la réglementation dans la mesure où la régulation vise à appliquer le cadre législatif, fournir des avis sur les décisions des pouvoirs publics et les comportements des acteurs du marché, et enfin collecter l'information statistique pertinente sur le secteur en question. La réglementation, quant à elle, consiste en l'établissement du cadre juridique dans lequel doit s'exercer la régulation. Libéraliser les services publics ne signifie donc pas déréglementer et encore moins déréguler. $\mathrm{Au}$ contraire, la régulation et l'action réglementaire sont encore plus nécessaires pour veiller au bon fonctionnement du secteur libéralisé. Si le rôle de l'État en matière de réglementation n'est pas discuté, il l'est en revanche en matière de régulation, notamment s'agissant de secteurs économiques, comme le secteur ferroviaire, confrontés à la concurrence mais encore marqués par la présence de l'État.

\subsection{Les conditions d'une régulation réussie}

La question de la «bonne » régulation est essentielle à la réussite du processus d'ouverture à la concurrence. Une étude réalisée pour les chemins de fer allemands établit, en effet, une corrélation entre une régulation efficace et l'ouverture du marché ferroviaire (Henry, 1999). Mais, la question de la «bonne » régulation est complexe comme en attestent les réponses différentes mais, non opposées, qui ont été apportées (tableau 2). 
Tableau 1 : Fonctions essentielles de la régulation d'une industrie de réseau libéralisée

\begin{tabular}{|c|c|c|c|}
\hline $\begin{array}{c}\text { Type de } \\
\text { régulation }\end{array}$ & Fonction & Contenu & Exemples \\
\hline Technique & $\begin{array}{l}\text { Régulation } \\
\text { technique }\end{array}$ & $\begin{array}{l}\text { Définition et contrôle } \\
\text { des normes techniques } \\
\text { - Sécurité des instalations } \\
\text { et matériel }\end{array}$ & $\begin{array}{l}\text { Normes de voltage, } \\
\text { système de sécurité } \\
\text { ferroviaire, etc ; }\end{array}$ \\
\hline \multirow[t]{2}{*}{ Économique } & $\begin{array}{l}\text { Régulation du } \\
\text { marché }\end{array}$ & $\begin{array}{l}\text { - Introduction } \\
\text { de la concurrence } \\
\text { - Accès des tiers au réseau } \\
\text { - Régulation des monopoles } \\
\text { naturels } \\
\text { - Surveillance des prix }\end{array}$ & $\begin{array}{l}\text { Fixation de la taxe } \\
\text { de raccordement au } \\
\text { réseau électrique, } \\
\text { règles d'accès } \\
\text { au réseau ferroviaire }\end{array}$ \\
\hline & $\begin{array}{l}\text { Régulation } \\
\text { de la concurrence }\end{array}$ & $\begin{array}{l}\text { - Régulation anti-trust } \\
\text { - Abus de position dominante } \\
\text { - Comportement anti- } \\
\text { concurrentiel }\end{array}$ & $\begin{array}{l}\text { Interdiction des } \\
\text { accords cartellaires } \\
\text { sur les tarifs } \\
\text { téléphoniques }\end{array}$ \\
\hline \multirow[t]{2}{*}{ Sociopolitique } & $\begin{array}{l}\text { Mise en œuvre } \\
\text { des politiques } \\
\text { publiques } \\
\text { connexes }\end{array}$ & $\begin{array}{l}\text { Poursuite des objectifs } \\
\text { de politiques publiques } \\
\text { connexes }\end{array}$ & $\begin{array}{l}\text { Économies } \\
\text { d'énergie, transfert } \\
\text { marchandises } \\
\text { sur le rail }\end{array}$ \\
\hline & $\begin{array}{l}\text { Régulation du } \\
\text { service public }\end{array}$ & $\begin{array}{l}\text { - Service universel } \\
\text { (Qualité, accès, prix) } \\
\text { - Sécurité }\end{array}$ & $\begin{array}{l}\text { Obligation de } \\
\text { raccordement aux } \\
\text { réseaux électriques } \\
\text { et téléphoniques }\end{array}$ \\
\hline
\end{tabular}

(En italique, les fonctions nouvelles de la régulation à la suite de la libéralisation)

Source : Genoux, 2004 : 190

Tableau 2 : Les critères de la « bonne » régulation

\begin{tabular}{|c|c|c|c|}
\hline Auteurs & Bergougnoux (2000) & Curien (2001) & Sauvé (2010) \\
\hline \multirow{4}{*}{ Critères } & - indépendance & - clarté des objectifs & - réponse à un besoin réel \\
\hline & - force : budget & - adéquation des moyens & $\begin{array}{l}\text { - définition précise } \\
\text { de la mission }\end{array}$ \\
\hline & $\begin{array}{l}\text { d'investigation et de } \\
\text { sanction... }\end{array}$ & $\begin{array}{l}\text { - importance des } \\
\text { pouvoirs }\end{array}$ & $\begin{array}{l}\text { - ligne de partage claire } \\
\text { entre l'instance et le } \\
\text { gouvernement }\end{array}$ \\
\hline & & $\begin{array}{l}\text { - transparence des } \\
\text { procédures } \\
\text { - indépendance }\end{array}$ & $\begin{array}{l}\text { - indépendance et force } \\
\text { suffisante }\end{array}$ \\
\hline
\end{tabular}

Comme le montre le tableau 2, les conditions d'une régulation réussie sont multiples. Néanmoins, au-delà de l'apparente multiplicité, une base commune de critères, à l'aune desquels la régulation doit être jugée, peut être repérée. L'indépendance et la force sont, 
en effet, des critères communs aux trois analyses d'une régulation efficace présentées ici. C'est toutefois le critère de l'indépendance qui apparaît comme supérieur. Une instance de régulation dotée de pouvoirs importants mais privée d'indépendance serait non seulement peu crédible mais, en plus, une menace pour le processus de libéralisation.

Le problème de l'indépendance du régulateur sectoriel est ancien. Il émerge aux États-Unis où les agences de régulation (regulation agencies), qui existent depuis le début du $\mathrm{XX}^{\mathrm{e}}$ siècle, doivent affirmer leur indépendance vis-à-vis du pouvoir politique, au niveau fédéral et fédéré, mais aussi des entreprises du marché régulé. Des mesures visant à rendre ces agences plus indépendantes sont depuis lors régulièrement étudiées dans les revues de droit et de science politique américaine (Fesler, 1940 ; Robinson, 1971 ; Bernstein, 1972 ; Morrison, 1988).

Plus généralement, l'indépendance, dans les États de droit fondés sur le principe de la séparation des pouvoirs, est rapidement apparue comme un impératif en priorité pour le pouvoir judiciaire. Au XVIII' ${ }^{\mathrm{e}}$ siècle, Montesquieu $(1748$, xi, 6) n'affirmait-il pas déjà qu' "il n'y a point (...) de liberté si la puissance de juger n'est pas séparée de la puissance législative et de l'exécutrice ». La thématique de l'indépendance, très présente chez les juristes, a naturellement été reprise par la littérature économique s'intéressant à la réglementation, celle-ci étant envisagée comme une solution aux défaillances du marché (Pigou, 1920 ; Coase, 1960) ${ }^{6}$. Au sein des développements récents de cette littérature (Djankov et al., 2003 ; Glaeser et Shleifer, 2003), le thème de l'indépendance est, en particulier, étudié par la théorie de la capture. L'objet principal de cette nouvelle théorie est de formaliser la capture des décisions publiques par les groupes de pression, en d'autres termes la réciprocité entre les décideurs politiques et les groupes de pression. En France, cette formalisation est entreprise principalement par Laffont et Tirole (1991), qui, au moyen de la théorie du principal et de l'agent, illustrent l'idée selon laquelle les enjeux des groupes de pression doivent être réduits afin de ne pas corrompre la réglementation.

En raison de leur récurrence dans les interprétations de la «bonne » régulation, ce sont les critères de l'indépendance et de la force que nous retiendrons également pour évaluer l'ARAF ainsi que l'instance qui l'a précédée.

\section{La régulation dans le domaine ferroviaire}

Mobilisant les nouvelles approches de la concurrence et notamment la théorie des marchés contestables (Baumol, Panzar et Willig, 1982), la Commission européenne définit

\footnotetext{
${ }^{6}$ Précisons que cette théorie renferme deux conceptions opposées du régulateur : la première l'envisage comme un agent entièrement préoccupé par la maximisation du bien-être social ; la seconde comme un agent vénal, soumis à l'influence de groupe de pression. C'est cette seconde conception du régulateur que la nouvelle économie de la réglementation reprend. La particularité de cette théorie économique est qu'elle a bénéficié du double apport de la science politique et de l'économie politique en matière d'organisation de l'État et du processus des décisions publiques. Elle a ainsi repris cette vision de la capture de la vie publique par les groupes de pressions qui a été développée par les spécialistes de sciences politiques (Bentley, Truman) et par les économistes des écoles de Chicago (Stigler, Becker) et de Virginie (Buchanan, Tullock) (voir Tirole, 1990 :307). En résumé, ce qui distingue fondamentalement la nouvelle économie de la réglementation de "l'ancienne », c'est son rapport à l'hypothèse de la bienfaisance de l'État. Alors que cette hypothèse est maintenue par une partie de l'ancienne économie de la réglementation, elle est totalement levée par la nouvelle économie de la réglementation.
} 
les modalités de mise en œuvre de la libéralisation reposant sur une séparation entre les activités liées au réseau, qui présentent des caractéristiques de rendements croissants, et les autres activités relevant de l'exploitation et des services.

\subsection{La transposition des directives européennes de libéralisation en France}

La directive européenne 440 de $1991^{7}$ stipule ainsi que « les États membres prennent les mesures nécessaires pour assurer, sur le plan de la comptabilité, la séparation des activités relatives à l'exploitation des services de transport de celles relatives à la gestion de l'infrastructure ferroviaire » (art. 6-1). La directive 12 de $2001^{8}$ précise, pour sa part, que « les États membres peuvent, en outre, prévoir que cette séparation comporte des divisions organiques distinctes au sein d'une même entreprise ou que la gestion de l'infrastructure est assurée par une entité distincte » (art. 6-2). C'est ce qu'a fait la France en choisissant dès 1997 de créer une entité distincte, Réseau Ferré de France (RFF), pour assurer la gestion de l'infrastructure ferroviaire.

Trois paquets de directives, adoptés en 2001, 2004 et 2007 marquent les grandes étapes de la libéralisation, en ouvrant progressivement à la concurrence d'abord sur des segments de marché du fret ferroviaire, jusqu'à l'ouverture totale du fret ferroviaire en 2006, puis plus récemment sur le segment du transport international de passagers. Mais cette libéralisation nécessite en parallèle un certain nombre de réglementations complémentaires portant sur l'interdiction des subventions croisées, la définition des règles et des charges d'accès, la compatibilité des matériels et des réseaux, la sécurité et les obligations de service public. La Commission européenne fixe ainsi en 1995 dans des directives « filles » de la directive 91/440/CEE les principes d'équité et de non discrimination dans les conditions d'attribution des licences d'accès à l'infrastructure ou de tarification de l'usage de cette infrastructure. Le processus d'ouverture à la concurrence du secteur ferroviaire se traduit par un « véritable mouvement de re-réglementation » comme le souligne le rapport fait au nom de la commission des Affaires économiques par le sénateur Grignon (2009 : 11).

L'Union européenne insiste également dès 2001 sur la nécessité d'un contrôle de la bonne mise en œuvre de la concurrence dans le secteur ferroviaire qui reposerait sur un organisme de contrôle, qualifié en anglais de « regulatory body $»^{9}$. Il s'agit notamment de s'assurer de la bonne mise en œuvre de la concurrence et du respect des principes d'un traitement équitable et non discriminatoire entre tous les exploitants.

La France transpose ces exigences en créant la Mission de Contrôle des Activités Ferroviaires

${ }^{7}$ Directive 91/440/CEE du Conseil sur le développement des chemins de fer communautaires du 29 juillet 1991, JOCE L 237/25 du 4 août 1991.

${ }^{8}$ Directive 2001/12/CE du Parlement européen et du Conseil du 26 février 2001 modifiant la directive 91/440/ CEE du Conseil relative au développement de chemins de fer communautaires, JOCE L 75 du 15 mars 2001.

9 Selon la directive 2001/14/CE : "Sans préjudice des dispositions de l'article 21, paragraphe 6, les États membres instituent un organisme de contrôle. Cet organisme, qui peut être le ministère chargé des questions de transports ou tout autre organisme, est indépendant des gestionnaires d'infrastructure, des organismes de tarification, des organismes de répartition et des candidats, sur le plan organisationnel, juridique, décisionnel et pour ce qui est décisions en matière financière. Cet organisme fonctionne conformément aux principes énoncés au présent article, les fonctions de recours et de contrôle pouvant être attribuées à des organismes séparés. Un candidat peut saisir cet organisme dès lors qu'il estime être victime d'un traitement inéquitable, d'une discrimination ou de tout autre préjudice, notamment pour introduire un recours contre les décisions prises par le gestionnaire de l'infrastructure ou, le cas échéant, par l'entreprise ferroviaire » (art. 30). 
(MCAF) en 2003, qui est placée auprès du ministre chargé des Transports. Mais la Commission Européenne, dans le cadre d'une procédure entamée en 2008 par une mise en demeure, fait valoir les lacunes de la France dans la mise en application des directives du «premier paquet ferroviaire» sur l'ouverture à la concurrence du marché européen du rail. Parmi celles relevées, figure notamment l'absence d'un organisme de régulation indépendant doté des pouvoirs nécessaires pour résoudre les problèmes de concurrence dans le secteur ferroviaire. Un courrier complémentaire a été adressé en juillet 2009 et un avis motivé en octobre 2009, auxquels la France a répondu. La loi 1503 de 2009 est un des éléments de réponse fournis afin de mieux répondre aux directives communautaires en matière de régulation, tout en mettant en œuvre la libéralisation des services de transport internationaux de voyageurs prévue par le troisième paquet ferroviaire 2004/2007. L'ARAF succède ainsi en 2009 à la MCAF. La Commission européenne a salué cette amélioration de la législation ferroviaire et a donc réduit la portée des procédures d'infraction, en reconnaissant que la France a institué un organe de régulation indépendant et doté des pouvoirs nécessaires. La France demeure néanmoins parmi les treize États membres contre lesquels la Commission a formé un recours devant la Cour de justice de l'UE en juin 2010 afin de régler les autres problèmes tenant à la tarification de l'accès à l'infrastructure et à l'indépendance des fonctions essentielles.

Entre temps, la France crée l'Établissement public de sécurité ferroviaire (EPSF) en 2006. Cette création est demandée par le deuxième paquet ferroviaire 2002/2004, qui dispose que « dans le nouveau cadre réglementaire commun pour la sécurité ferroviaire, il convient d'établir dans tous les États membres des autorités nationales chargées de réglementer et contrôler la sécurité ferroviaire » (directive 49 de $2004^{10}$ ).

Ainsi, le nouveau cadre de fonctionnement du système ferroviaire, dont l'objectif est d'instaurer la concurrence dans un secteur traditionnellement monopolistique, substitue au couple État/entreprise ferroviaire publique le quintet d'acteurs publics suivants :

- État;

- SNCF, entreprise ferroviaire;

- RFF, gestionnaire d'infrastructure;

- EPSF, établissement public de sécurité ferroviaire;

- ARAF, autorité de régulation des activités ferroviaires.

\subsection{La MCAF : une première mise en œuvre de la régulation peu concluante}

L'ARAF n'est pas la première instance de régulation ferroviaire en France. Elle a été précédée par la Mission de Contrôle des Activités Ferroviaires (MCAF) créée en 2003. Créée en vue de l'ouverture du service de transport ferroviaire de marchandises à la concurrence en mars 2006, la MCAF est dotée de plusieurs prérogatives dans ce secteur (encadré 1).

Composée de six membres (trois titulaires et trois suppléants membres, respectivement, du Conseil d'État, de la Cour des Comptes et du Conseil Général des Ponts et Chaussées), la MCAF est assistée dans sa tâche, par un agent titulaire du ministère des transports qui fait fonction de secrétaire général. Ses membres ne sont pas rémunérés et elle n'a ni budget ni crédits propres. L'absence d'autonomie financière est d'ailleurs un grief qu'adresse régulièrement la MCAF au ministre en charge des transports dans ses différents rapports

${ }^{10}$ Directive 2004/49/CE du Parlement européen et du Conseil du 29 avril 2004, JOUE L220 du 21 juin 2004. 
d'activité. Ces derniers insistent en outre sur la nécessité d'apporter des améliorations au fonctionnement de l'instance. Le rapport couvrant les activités de l'instance au cours de l'année 2006 souligne ainsi la nécessité de placer la MCAF comme destinataire direct des réclamations adressées au ministre.

Encadré 1 : Les compétences de la Mission de Contrôle des Activités Ferroviaires

La MCAF est déjà dotée de compétences étendues : en vertu de l'article 29 du décret 2003/194, la MCAF est chargée d'instruire les réclamations relatives aux sujets suivants (eux-mêmes décrits à l'article 28 du décret pré-cité) :

- le contenu du document de référence du réseau ;

- la procédure de répartition des capacités d'infrastructure et les décisions afférentes ;

- le système de tarification, ainsi que le niveau et la structure des redevances d'utilisation de l'infrastructure empruntée, en particulier au regard des dispositions mentionnées dans le décret $n^{\circ}$ 97-446 du 5 mai 1997 ;

- l'exercice du droit d'accès au réseau ;

- la fourniture des prestations et services de l'article 3 du décret 2003/194;

- la mise en œuvre des accords-cadres ainsi que des contrats d'utilisation de l'infrastructure.

Elle est aussi chargée d'assurer une mission générale d'observation des conditions d'accès au réseau ferré national. Elle peut, à ce titre, formuler toute recommandation utile au ministre.

La MCAF est en outre chargée d'instruire les demandes adressées au ministre au titre des dispositions prévues au $2^{\mathrm{e}}$ alinéa de l'article 25 du décret $\mathrm{n}^{\circ}$ 2006-1534 du 6 décembre 2006 : la MCAF, sur saisine du ministre chargé des Transports, donne son avis sur les différends opposant le délégataire à RFF ou à la $\mathrm{SNCF}$, portant sur l'application des règles relatives au droit d'accès à l'infrastructure.

Source : www.senat.fr/rap/l08-184/l08-1845

Mais le talon d'Achille de cette instance réside moins dans les pouvoirs qui lui ont été conférés que dans son indépendance. Comme le souligne le rapport Grignon au nom de la Commission des affaires économiques du Sénat ${ }^{11}$, le pouvoir de régulation appartient in fine au ministre des transports, la MCAF ayant pour mission de l'assister en instruisant les réclamations des entreprises et en lui adressant de simples avis consultatifs ou des recommandations. Seul le ministre peut, le cas échéant, édicter une sanction, ce qui affaiblit l'autorité de la MCAF. Son cadre formel influe sur son fonctionnement car le fait que la MCAF soit placée auprès du ministre l'entrave dans sa capacité à décider seule de publier telle ou telle recommandation sans avoir à en référer au ministre. La MCAF ne s'exprime que sous forme d'avis ou de recommandations au ministre. De plus, son statut de « service » du ministère ne prévoit pas pour ses membres de cas spécifiques d'incompatibilités ou susceptibles d'entraîner la révocabilité des mandats des membres de la commission ou de son président, notamment en cas de détention d'intérêt dans une entreprise du secteur ferroviaire. Le statut d'autorité administrative indépendante prévoit au contraire ces cas.

${ }^{11}$ Rapport n¹84 déposé le 28 janvier 2009, dans le cadre de la discussion sur le projet de loi ORTF. 
En termes de bilan, seules deux réclamations ont été soumises à la MCAF d'après les rapports d'activité établis jusqu'en 2007. La mission relevait d'ailleurs dans le rapport de 2007 que les entreprises ferroviaires hésitaient « à déposer des réclamations auprès de la mission de contrôle, en raison du manque de crédibilité de celle-ci, et par crainte de susciter de nouvelles difficultés dans leurs rapports avec la SNCF » (MCAF, 2008 : 12). Elle soulignait que "l'établissement d'une véritable autorité de régulation du transport ferroviaire en France, à l'image de celles créées lors de la libéralisation des marchés de l'énergie et des télécommunications » (MCAF, 2008 : 12) était une condition préalable aux recours soulignée par les opérateurs.

Le contrôle de l'État sur le régulateur crée donc un conflit d'intérêt entre, d'un côté, l'impartialité attendue de ce régulateur envers l'ensemble des opérateurs et, de l'autre, le souci de maintenir la santé financière de l'entreprise ferroviaire dont l'État a la tutelle. La convergence d'intérêts entre le ministère français des transports et la SNCF pose la question de l'indépendance d'un régulateur directement rattaché au ministère des transports. Face à ce risque de capture de la régulation, la Cour des Comptes (2008 :138) proposait en 2008 de transférer la gestion de l'infrastructure à une filiale de la SNCF afin qu'une partie des personnels de RFF intègre les équipes de la régulation, permettant ainsi de disposer d'une réelle contre-expertise externe à la SNCF.

\section{L'ARAF a-t-elle tiré les leçons du passé ?}

L'irruption récente de l'ARAF dans le paysage ferroviaire français permet-elle d'améliorer les modalités de régulation du secteur ferroviaire ? Pour répondre à cette question, nous examinerons l'ARAF à l'aune des deux précédents critères : l'indépendance et la force.

\subsection{L'ARAF, une autorité indépendante?}

Pour prévenir tout éventuel manque d'indépendance du régulateur en matière ferroviaire, le rapport Bergougnoux (2000 : 154) insiste sur la nécessaire distance du régulateur «aussi bien vis-à-vis de l'ensemble des acteurs du secteur, gestionnaire d'infrastructure et exploitants, français et étrangers, que vis-à-vis des pouvoirs publics eux-mêmes». L'article 20 de la loi n²009-1503 précise que «les membres et agents de l'Autorité de régulation des activités ferroviaires exercent leurs fonctions en toute impartialité, sans recevoir d'instruction du Gouvernement ni d'aucune institution, personne, entreprise ou organisme ». Cette séparation est mise en œuvre en mobilisant le statut juridique d'Autorité Administrative Indépendante, catégorie juridique qui, contrairement à la tradition administrative française, caractérise des institutions de l'État non soumises à l'autorité hiérarchique d'un ministre. L'ARAF a, en plus, l'autonomie financière et la personnalité morale. Pour autant, ce statut suffit-il à fournir une réelle indépendance à cette instance ? Cette question mérite d'être posée quand on examine les éléments suivants relatifs au fonctionnement de l'ARAF :

- les modalités de désignation des membres du collège ;

- les conditions d'exercice du mandat;

- l'autonomie financière. 


\section{Les modalités de désignation des membres du collège}

La direction de l'ARAF est exercée collégialement par sept membres. Les membres du collège sont nommés par décret, du président de la république pour le président de l'ARAF et du premier ministre pour les six autres membres (JORF $n^{\circ} 167$ du 22 juillet 2010, p. 13531 et JORF $n^{\circ} 167$ du 22 juillet 2010, p. 13531). Par ailleurs, une partie de ces membres est proposée respectivement par le Sénat, l'Assemblée Nationale et le Conseil Économique, Social et Environnemental. Ainsi, si l'ARAF n'appartient pas à la hiérarchie de l'administration et dispose d'un pouvoir propre, elle n'en entretient pas moins cependant de nombreuses relations avec l'administration et les institutions politiques, notamment dans les modalités de désignation de ses dirigeants. La diversité des modalités de nomination est toutefois censée éviter une emprise politique trop importante.

Envisageons à présent les liens entre les membres de l'ARAF et les acteurs du secteur ferroviaire. L'article 13 de la loi ORTF (Organisation et Régulation du Transport Ferroviaire de 2009) prévoit que les membres du collège ne doivent pas avoir d'intérêts, directs ou indirects, avec les entreprises du secteur ferroviaire. Or, même si ces intérêts ne sont plus avérés à l'heure actuelle, certains membres du collège ont occupé des fonctions importantes auprès des acteurs du secteur. Les flux de personnels entre l'autorité et l'industrie en question sont également illustratifs. Si les recrutements pour l'ARAF sont en cours, la technicité du monde ferroviaire pose la question de la réelle possibilité d'indépendance. Le développement de ces agences, qualifié d'agencification, est ainsi fortement corrélé à une technicisation des secteurs d'activité et à la participation de professionnels du secteur, laquelle est parfois considérée comme un gage d'efficacité et de légitimité accrue de l'action publique.

\section{Les conditions d'exercice du mandat}

Plusieurs dispositions ont été prises pour limiter la collusion entre les intérêts des membres de l'ARAF et ceux des pouvoirs publics. Ainsi, les membres du collège ne peuvent détenir ni mandat électif, ni emploi public pendant leurs fonctions au sein de l'ARAF (art. 13 de la loi $n^{\circ}$ 2009-1503). Cependant, l'Autorité peut employer des magistrats et des fonctionnaires (art. 19). En outre, les mandats des membres du collège sont irrévocables (art. 13). Mais, là encore, il convient de noter que les mandats des six membres du collège - à l'exception de celui du président - sont renouvelés par tiers tous les deux ans (art. 12-I). La durée du mandat des membres désignés par les présidents des assemblées parlementaires et du Conseil Économique, Social et Environnemental est ainsi fixée par tirage au sort à deux, quatre et six ans. La durée du mandat des trois autres membres est également fixée par tirage au sort à deux, quatre et six ans. Les modalités d'évolution pour la durée de leur mandat incombent, en outre, aux Ministres chargés des Transports et du Budget (art. 19). Or, il est admis que les garanties statutaires accordées aux membres de ces autorités gagneraient à être renforcées car elles sont importantes pour assurer l'indépendance de la structure.

Par ailleurs, les statuts initiaux de l'ARAF précisaient que son président ne pouvait exercer aucun mandat politique. Mais, un amendement a introduit la possibilité que le futur président exerce un mandat municipal. Ainsi, Pierre Cardo, qui a été nommé le 20 juillet 2010 à la tête de l'ARAF, peut demeurer Président de la communauté d'agglomération des Deux Rives de la Seine et ainsi suivre le projet du Grand Paris. 


\section{L'autonomie financière}

L'ARAF est financée par les entreprises ferroviaires utilisant le réseau comme, par exemple, la SNCF (voir art. 21-II de la loi n²009-1503) ${ }^{12}$. Le budget est alimenté par un droit fixe correspondant à $0,37 \%$ des péages versés à RFF par les entreprises ferroviaires. L'ARAF est ainsi financièrement indépendante par rapport aux pouvoirs publics, puisque son financement ne provient pas du budget de l'État, mais dépendante vis-à-vis des exploitants. Or, si la dépendance par rapport à l'État est souvent dénoncée, il convient d'être également vigilant au risque de dépendance par rapport aux acteurs du secteur à réguler. Toutefois, la loi de finances 2012, qui limite son budget à 11 millions d'euros, vient questionner l'indépendance financière de l'ARAF. Cette restriction, qui fait suite à un amendement gouvernemental voté par les députés en novembre 2011, assimile l'ARAF aux agences gouvernementales, lesquelles sont soumises à des restrictions budgétaires.

\subsection{L'ARAF, une autorité puissante, voire trop puissante ?}

La force d'une instance de régulation peut se mesurer de différentes manières. Il faut, entre autre, qu'elle dispose de moyens humains et financiers conséquents et de pouvoirs de sanction.

\section{Des moyens conséquents}

Par rapport à la MCAF, l'ARAF dispose de moyens humains plus grands. Tout d'abord, l'ARAF a un exécutif collégial composé de sept membres alors que l'exécutif de la MCAF n'était composé que de quatre membres : un président par un membre du Conseil d'État, un membre de la Cour des comptes, un membre du Conseil général des Ponts et Chaussées ainsi qu'un suppléant (art.1 de l'arrêté du 6 mai 2003 ${ }^{13}$ ). Ensuite, l'ARAF est dotée de 52 emplois équivalent-temps-plein. Enfin, l'ARAF peut, dans certaines situations, mobiliser des agents contractuels (art. 19 de la loi n²009-1503) ou des experts (art. 22). Les personnels sont affectés dans le service des affaires générales ou dans les trois directions opérationnelles : direction des affaires juridiques, direction de l'accès au réseau en charge des aspects économiques et techniques, direction de l'audit comptable. Les profils s'adressent à des experts du domaine ferroviaire, mais aussi de l'économie des transports, de l'audit financier et du droit. Les agents proviennent d'univers professionnels divers (entreprises, cabinets d'audit, universités, autres régulateurs...). Enfin, l'ARAF a de larges pouvoirs d'investigation. Elle est ainsi autorisée à accéder à la comptabilité et aux locaux des gestionnaires d'infrastructure et des entreprises ferroviaires. Elle peut également recueillir toutes les informations qui peuvent lui être utiles (art. 22).

\section{De réels pouvoirs de sanction}

Lorsqu'un gestionnaire d'infrastructure ou une entreprise ferroviaire ne s'est pas conformé à la mise en demeure de l'ARAF dans le délai imparti, celle-ci peut recourir aux sanctions suivantes : «Une interdiction temporaire d'accès à tout ou partie du réseau ferroviaire pour une durée n'excédant pas un an ; une sanction pécuniaire, dont le montant est proportionné à la gravité du manquement, à la situation de l'intéressé...»(art. 17-I 1º). Ces pouvoirs

\footnotetext{
12 Notons que c'est le Sénat qui a conféré à I'ARAF une personnalité morale propre lui permettant d'être financée par des ressources propres, alors que le texte initial prévoyait qu'elle demeure juridiquement intégrée à l'État.

${ }^{13}$ Arrêté du 6 mai 2003 fixant les modalités de fonctionnement de la mission de contrôle des activités ferroviaires (www.legifrance.gouv.fr).
} 
de sanction doivent également être délimités par rapport à ceux du juge. C'est notamment au juge seul que revient le droit de lire et d'interpréter le droit.

\section{Des missions importantes}

Le texte législatif relatif à la création de l'ARAF indique clairement les missions de cette instance. Le rapport dirigé par Bergougnoux (2000 : 156) ajoute que l'instance doit pouvoir agir au moins dans les quatre directions suivantes :

- Le contrôle du gestionnaire d'infrastructures. L'ARAF a pour mission générale d'observer les conditions d'accès au réseau ferroviaire (art. 11). Elle peut s'opposer aux tarifs négociés par le gestionnaire d'infrastructure (art. 15-II). Le 25 janvier 2012, l'ARAF a ainsi demandé la mise en place d'un barème de péages prévisionnel et pluriannuel à RFF.

- La production d'information sur la situation et le développement du secteur. L'Autorité émet divers avis : sur la fixation des redevances d'infrastructure (art. 15-V), sur certaines nominations (art. 15-IX)... C'est la Haute Assemblée qui a contribué à étendre le champ d'intervention de l'ARAF en lui reconnaissant la possibilité d'une part de donner son avis sur le document de référence du réseau, d'autre part de veiller à l'équité des politiques tarifaires (article 8). Ceci constitue pour l'instant l'essentiel des sept avis rendus par l'ARAF.

- La vérification de l'absence de subventions croisées. L'ARAF veille à ce que les règles de la séparation comptable, celles d'imputation et les périmètres comptables ne permettent aucune discrimination, subvention croisée ou distorsion de concurrence (art. 15-IV). Une seule décision de ce type a été rendue en octobre 2011 concernant la tenue de comptes séparés pour l'activité de gestion des gares par la SNCF. La décision de l'ARAF n'approuvait alors pas les règles proposées par la SNCF et l'invitait à en soumettre de nouvelles.

- L'instruction des demandes de licences ferroviaires. L'une des premières missions de l'instance de régulation est de veiller à ce que les conditions d'accès au réseau ferroviaire par les entreprises ferroviaires n'entravent pas le développement de la concurrence (art. 11). Une dizaine de règlements a ainsi été produite par l'ARAF concernant cinq concurrents - EuroCargo Rail, Novatrans, Froid Combi, Europorte Channel - de la SNCF et/ou du RFF.

À ces quatre missions déjà importantes s'ajoutent d'autres pouvoirs. L'Autorité émet ainsi des recommandations en matière de loi ou de règlement et peut être associée aux négociations internationales et européennes (art. 11). Depuis sa création, l'ARAF a ainsi formulé sept avis sur des projets de décret ou d'arrêté.

Elle peut également avoir un pouvoir réglementaire supplétif. En effet, elle peut, dans certaines situations, préciser des règles de nature technique ou encore comptable (art. 14). Cette mission nécessite que la répartition des compétences entre l'autorité et le gouvernement soit clairement explicitée, notamment dans les textes constitutifs. Cet enjeu est lié à celui de l'indépendance. L'inscription dès l'origine dans les textes des compétences respectives 
réduit les risques de présence d'un commissaire de gouvernement au sein des autorités administratives, solution parfois retenue afin de tenir informé le ministre des décisions prises par l'autorité et de faire valoir en retour la position du gouvernement.

Toutefois, l'étendue des missions évoquées ci-dessus semble encore insuffisante, si l'on en croit l'Autorité de la concurrence, dans son avis n¹1-A-15 du 29 septembre 2011 : «l'expérience tirée de l'ouverture à la concurrence d'autres industries de réseaux monopolistiques appelle dans ce cadre à renforcer de manière très significative les pouvoirs du régulateur sectoriel. En l'état actuel des textes, l'ARAF ne paraît pas en mesure de répondre pleinement aux objectifs de la régulation sectorielle, que ce soit en termes de signal économique envoyé ou de prévention des pratiques anticoncurrentielles. La situation actuelle est ainsi tout à fait préoccupante en termes de sécurité tant juridique qu'économique ». Les carences portent plus spécifiquement sur le traitement des coûts d'accès aux facilités essentielles. En l'état, l'opérateur historique détermine seul ces coûts d'accès et les services rendus sur ces installations, puisque l'ARAF ne dispose pas du pouvoir de contrôle tarifaire ex ante.

\section{Des contre-pouvoirs insuffisants}

Il est surprenant de constater que peu de pouvoirs susceptibles de s'opposer aux nombreux et larges pouvoirs de l'ARAF ont été prévus par les textes de loi. Le décret du $1^{\text {er }}$ septembre 2010 précise les conditions de recours. Certes, l'Autorité est soumise au contrôle de la Cour des Comptes (art. 21) et doit établir chaque année un rapport d'activité qui est rendu public (art. 26). Mais, hormis ces dispositions, les actions comme les membres de l'ARAF ne semblent pas subir de véritable contrôle. Ainsi, certaines décisions (celles prises au titre de l'art. 16-II) peuvent être contestées par la Cour d'appel de Paris dans un délai d'un mois à compter de leur notification. Mais, ce recours n'est pas suspensif. Seul le recours devant le Conseil d'État contre les sanctions pécuniaires décidées par l'ARAF a un caractère suspensif (art. 17-II). En outre, ses membres ne doivent pas rendre compte de leur action devant les assemblées parlementaires. La création dans chaque Assemblée d'une délégation chargée spécifiquement du suivi des autorités administratives indépendantes, comme l'ARAF, constituerait une réponse à ce manque. Mais, une telle délégation se verrait remettre une charge de travail conséquente, en raison de la multiplication ces dernières années de ces Autorités, sauf à raisonner comme le suggère Frison-Roche (2004) en terme d' « interrégulation».

\section{Conclusion}

Des conditions juridiques visant à instituer l'indépendance de l'ARAF - pluralisme des sources de nomination, non-cumul des fonctions, autonomie financière...- et à lui conférer un réel pouvoir ont été prises. Il est certes difficile d'évaluer l'influence des statuts de l'ARAF sur son fonctionnement, en raison de la jeunesse de la structure. Depuis sa création, très récente, l'ARAF a rendu neuf avis, et traité deux différends. La possibilité même d'indépendance de la structure peut néanmoins être questionnée, en raison des compétences spécifiques nécessaires à l'exercice de ses missions et de l'origine qu'elles supposent. La force de la structure peut également être interrogée au regard des asymétries d'informations relevées par l'autorité de la concurrence dans la tarification des facilités essentielles. 
Outre la faisabilité de la mise en place d'une instance de régulation fonctionnant de manière indépendante, c'est aussi le bien-fondé des instances de régulation au regard de leurs coûts qui est en question. Ainsi, la multiplication de ces instances à l'échelle européenne entre en contradiction avec la logique de rentabilité économique de la Commission européenne. Le coût généré par ces instances se justifie d'autant moins qu'elles ont seulement pour mission d'aider l'ouverture à la concurrence de secteurs autrefois gérés par des monopoles nationaux intégrés. Or, pourquoi créer des structures permanentes pour exercer des fonctions supposées transitoires ? Ne sont-elles pas alors la traduction des limites du marché ?

La transformation du rôle de l'État dans les activités de réseaux mérite, en outre, d'être examinée de près. Celle-ci ne touche, en effet, que certains domaines de ces activités. Ainsi, la sécurité du transport ferroviaire est toujours gérée par l'État. La France n'est pas une exception en la matière puisque, par exemple, en Grande-Bretagne, la privatisation de British Rail n'a pas entrainé celle de la British Transport Police (Hamelin et Spenlehauer, 2007). Ce statu quo institutionnel ne révèle-t-il pas également les limites de la logique du marché ? Il y a plus de deux siècles, Adam Smith, pourtant considéré aujourd'hui comme le « père » du libéralisme économique anglais, ne remettait-il pas au souverain le devoir de police, à côté de celui de justice et de travaux publics ?

\section{Remerciements}

Les auteurs remercient les deux rapporteurs anonymes pour leurs avis instructifs. Elles restent évidemment seules responsables des éventuelles erreurs ou omissions contenues dans l'article.

\section{Bibliographie}

AGHION P., CAGE J., (2010). Le retour de l'État régulateur, Le Monde, 05 juillet.

BAUBY P., (2002). L'Europe des services publics : entre libéralisation, modernisation, régulation, évaluation, Politiques et Management Public 20 (1), 15-30.

BAUMOL W.J., PANZAR J.C. and WILLIG R.D. (1982). Contestable Markets and the Theory of Industry Structure, Harcourt Brace Jovanovich, New York.

BERGOUGNOUX J. (2000). Services publics en réseau : perspectives de concurrence et nouvelles régulations, La documentation française, Paris.

BERNSTEIN, M.H. (1972). Independent Regulatory Agencies: A Perspective on Their Reform, Annals of the American Academy of Political and Social Science 400, 14-26.

COASE R. (1960). The Problem of Social Cost, Journal of Law and Economics 3, 1-44.

COMBES P.-P., JULIEN B. et SALANIE B. (1997). La réglementation des monopoles naturels, In PERROT A. (ed.). Réglementation et Concurrence, Économica, Paris, 9-30.

COUR DES COMPTES (2008). Rapport public thématique Le réseau ferroviaire. Une réforme inachevée, une stratégie incertaine, (www.ccomptes.fr/fr/CC/documents/RPT/RPT-reseauFerroviaire.pdf).

CURIEN N. (2001). La libéralisation des télécommunications en Europe, Flu 2-3 (44), 28-35.

DJANKOV S., LA PORTA R., LOPEZ-DE-SILANES F., SHLEIFER A. (2003). The Regulation of Entry, Quarterly Journal of Economy 117 (1), 1-37.

DRUMAUX A. et GOETHALS C. (2006). Qui profite de l'ouverture à la concurrence des monopoles publics ? Politiques et Management Public 24 (3), 33-56. 
FESLER J.W. (1940). Independence of State Regulatory Agencies, The American Political Science Review 34 (5), 935-947.

FRISON-ROCHE M.-A. (2004). Les nouveaux chemins de la régulation, Revue française d'administration publique 109, 53-63.

GENOUD C. (2004). Libéralisation et régulation des industries de réseau : diversité dans la convergence ? Revue Internationale de Politique Comparée 11 (2), 187-204.

GLAESER E.L., SHLEIFER, A. (2003). The Rise of Regulatory State, Journal of Economic Literature 41 (2), 401-425

GRIGNON F. (2009)., Rapport au Sénat $n^{\circ} 184$ fait au nom de la Commission des Affaires Economiques, sur le projet de loi relatif à l'organisation et à la régulation des transports ferroviaires et guidés et portant diverses dispositions relatives au transport (www.senat.fr/rap/108-184/108-184.html).

HAMELIN F. et SPENLEHAUER V. (2007). Les faux-semblants de la privatisation en Grande-Bretagne. L'exemple de la police des chemins de fer, Politiques et Management Public 25 (3), 115-130.

HENRY C. (1999). Étude comparative des conditions et instruments de la régulation économique de services publics en réseaux, École Polytechnique, 199 p.

LAFFONT J.-J., TIROLE J. (1991). The Politics of Government Decision-Making: A Theory of Regulatory Capture, The Quarterly Journal of Economics 106 (4), 1089-1127.

LANDIER A. et THESMAR D. (2010). La société translucide, Fayard, Paris.

LEVEQUE F. (2004). Économie de la réglementation, La découverte, Paris.

MCAF (2008). Rapport d'activité 2007 (www.assorail.fr/userfiles/webeditor/files/.../2007_MCAF-Rapport.pdf).

MONTESQUIEU C.-L. de SECONDAT, Baron de (1979). De l'esprit des lois, 2 vols., GF-Flammarion, Paris.

MORRISON, A.B. (1988). How Independent are Independent Regulatory Agencies ? Duke Law Journal 37 $(2 / 3), 252-256$.

OCDE (1988). Déréglementation et concurrence dans le transport aérien, OCDE, Paris.

PIGOU A.C. (1920). The Economics of Welfare, MacMillan \& Co, London.

POWELL E.R. (1997). Les référentiels normatifs de l'action publique en France et en Grande-Bretagne : les transports ferroviaires dans le contexte communautaire (1965-1995), Politiques et Management Public 15 (1), 97-116.

REYNOLDS-FEIGHAN A.J. (1992). The effects of Deregulation on U.S. Air Networks, Springer-Verlag, Berlin. ROBINSON, G.O. (1971). On Reorganizing the Independent Regulatory Agencies, Virginia Law Review 57 (6), 947-995.

SAUVE J.-M. (2010). Intervention relative aux autorités administratives indépendantes, audition dans le cadre de la mission mise en place par le comité d'évaluation et de contrôle des politiques publiques sur les autorités administratives indépendantes (www.conseil-etat.fr/cde/media/document/COLLOQUE/ intervention_aai_11022010.pdf).

SAVY M. (2010). Les directives européennes n'impliquent ni le démantèlement ni la privatisation de la SNCF, Euractiv.fr, (www.euractiv.fr/economie-finance/interview/2010/04/06/directives-europeennes-n-impliquentni-demantelement-ni-privatisation-sncf_66185).

TIROLE J. (1990). L'économie politique de la réglementation, L'Actualité économique, Revue d'analyse économique 66 (3), 305-318.

TOUWAIDE G. (2006). La déréglementation du marché du transport aérien régulier en Europe, mémoire, Université Libre de Bruxelles. 